\title{
Calculating the Fuzzy Project Network Critical Path
}

\author{
N. Shahsavari Pour, S. Zeynali, M. Pour Kheradmand \\ Department of industrial Engineering, Science and Research branch, Islamic Azad \\ University, Kerman, Iran. \\ Shahsavari_n@alum.sharif.edu \\ Department of industrial Engineering, Science and Research branch, Islamic Azad \\ University, Kerman, Iran \\ samira.zeinali@gmail.com \\ Department of industrial Engineering, Science and Research branch, Islamic Azad \\ University, Kerman, Iran \\ Mansour_Kheradmand@yahoo.com
}

\begin{abstract}
A project network consists of various activities. To determine the length of project time and the amount of the needed sources, the time of project completion must correctly and exactly be calculated, so the critical path is calculated. The activities on this path have no floating (variation). It means that there is no delay on these activities. As a result the calculation of the critical path in a project network has a special importance. In this paper a simple method for calculation the critical path is proposed. Assignment an exact time on any activity in real world is not correct; So the fuzzy and uncertainty theories are used to assigned a length of time on any activities. In the present study the trapezoidal fuzzy numbers are assigned to the length of activity time, and the total time of the project is also a fuzzy number. In addition, to compare the fuzzy numbers, ranking of fuzzy numbers are used. Finally a practical example will show the efficiency of the method.
\end{abstract}

Keywords: critical path method, fuzzy theory, floating, fuzzy number ranking 


\section{Introduction}

A project network consists of various activities. Each activity has a special completion time and logical order. In this way that any activity is done at a time that all activities before that are done. And also each activity has a special floating. It means that if the time of completion is delayed up to the amount of variation, no delay is seen in the all of project. As a result the project does not have any delay cost, and the project will be finished on time. However, the critical path in a project is the path that takes the longest completion time and determines the project completion time. All the activities on this path have variation of zero. It means that any delay in doing each of these activities leads to a delay in completion of the project and in accessibility to the time completion of the project. Assigning an exact number on activities increase the probability of error in calculations; therefore to determine the real time of activities the fuzzy theory is used. This method was proposed by professor Lotfizadeh which was used in various fields. In this study it is also used. The completion time has been determined by a group of specialist familiar with the project based on linguistic variable and changing them into fuzzy numbers.Chanas, Zielinski[1] presented a sense of being critical in a network, Dubois[2], presented an innovative method for calculation of various sets with a most delayed activity in start and variation. Also Zielinski [3] used the multi nominal algorithm to determine the most delayed start-time in a network, Feng, Jing [4] Proposed the fuzzy critical path which a-cut was used. Shahsavari pour, Modarres, Aryanejad, Tavakoli Moghadam[5], Calculated the project network critical path in uncertainty condition introducing FNR algorithm To compare the fuzzy numbers. In the present study a simple method based on random numbers to generate paths was used. And for compare fuzzy numbers a method called D- distance [6] will be utilized.

\section{Preliminary Notes}

In this problem, the project network is considered as a matrix (node-node matrix), $\mathrm{A}_{\mathrm{n} \times \mathrm{n}}=\left[a_{i j}\right]$ that $\mathrm{n}$ is the number of nodes.

Entry $a_{i j}$ is about $\mathrm{i}$ row and $\mathrm{j}$ column of the matrix of the network that is read as:

$$
a_{i j}=\left\{\begin{array}{cc}
1 & \text { if node } \mathrm{i} \text { starts } \operatorname{arc} \mathrm{j} \\
-1 & \text { if node } \mathrm{i} \text { ends } \operatorname{arc} \mathrm{j} \\
0 & \text { otherwise }
\end{array}\right\}
$$


If the connection is from i node to $\mathrm{j}$ node, there will be $a_{i j}=1$

If the connection is from $\mathrm{j}$ node to $\mathrm{i}$ node, there will be $a_{i j}=-1$

Otherwise it equals to zero.

In this study the number of paths in the project network will be calculated first, and then a special algorithm to find the project paths will be used. After creating all paths, the completion time of each path will be calculated. And based on the comparison of fuzzy numbers the path which has the most time is the critical path.

\section{2-1. The calculation of the number of paths in the project network}

To calculation the number of network paths node-to-node matrix as below is considered.

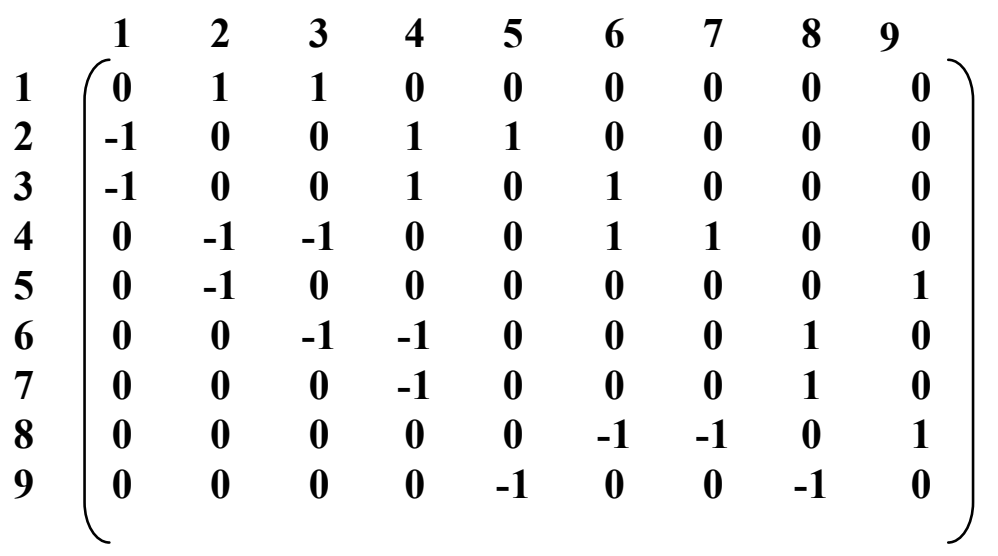

Any of these items of the matrix shows a connection between two nodes. To find the number of paths, in the mentioned matrix the only item related to $i<=j$ is considered and it is meant that the only 0, 1 from the mentioned matrix are calculated and the rest are not. Therefore the $M_{i j}^{1}$ matrix is defined as:

$$
\left.\begin{array}{llllllllll}
\mathbf{1} & \mathbf{1} & \mathbf{2} & \mathbf{3} & \mathbf{4} & \mathbf{5} & \mathbf{6} & \mathbf{7} & \mathbf{8} & \mathbf{9} \\
\mathbf{2} & \mathbf{0} & \mathbf{1} & \mathbf{1} & \mathbf{0} & \mathbf{0} & \mathbf{0} & \mathbf{0} & \mathbf{0} & \mathbf{0} \\
\mathbf{3} & \mathbf{0} & \mathbf{0} & \mathbf{1} & \mathbf{1} & \mathbf{0} & \mathbf{0} & \mathbf{0} & \mathbf{0} \\
\mathbf{4} & \mathbf{0} & \mathbf{0} & \mathbf{1} & \mathbf{0} & \mathbf{1} & \mathbf{0} & \mathbf{0} & \mathbf{0} \\
\mathbf{5} & \mathbf{0} & \mathbf{0} & \mathbf{0} & \mathbf{0} & \mathbf{0} & \mathbf{1} & \mathbf{1} & \mathbf{0} & \mathbf{0} \\
\mathbf{6} & \mathbf{0} & \mathbf{0} & \mathbf{0} & \mathbf{0} & \mathbf{0} & \mathbf{0} & \mathbf{0} & \mathbf{1} \\
\mathbf{7} & \mathbf{0} & \mathbf{0} & \mathbf{0} & \mathbf{0} & \mathbf{0} & \mathbf{0} & \mathbf{0} & \mathbf{1} & \mathbf{0} \\
\mathbf{8} & \mathbf{0} & \mathbf{0} & \mathbf{0} & \mathbf{0} & \mathbf{0} & \mathbf{0} & \mathbf{0} & \mathbf{1} & \mathbf{0} \\
\mathbf{9} & \mathbf{0} & \mathbf{0} & \mathbf{0} & \mathbf{0} & \mathbf{0} & \mathbf{0} & \mathbf{0} & \mathbf{0} & \mathbf{1} \\
& & & & & & & & &
\end{array}\right)
$$


Any of the items of the matrix shows the relations between two nodes. For example $M_{19}^{1}$ shows the number of paths as long as 1 from the first node to the last node. The purpose is to find the number of paths with the length of $(1,2$, $3 . . n$ ) from the first node to the last. Having found the number of paths, they will be added up, and the total number will be calculated. So, $M_{i j}^{1}$ matrix shows the number of paths with 1 length from $\mathrm{i}$ node to $\mathrm{j}$ node in the matrix. $M_{i j}^{2}$ Shows the number of paths with length 2 from node $\mathrm{i}$ to $\mathrm{j}$ node in the network and is calculated as:

$M_{i j}^{1}=$ matrix consist of Paths with length of 1 from $\mathrm{i}$ node to $\mathrm{j}$ node.

$M_{i j}^{2}=$ matrix consist of Paths with length of 2 from i node to $\mathrm{j}$ node.

$M_{i j}^{n}=$ matrix consist of paths with length of $\mathrm{n}$ from node $\mathrm{i}$ to $\mathrm{j}$ node.

$M_{i j}^{n}=M_{i j}^{n-1} * M_{i j}^{1}$

$\mathrm{n}$ is equal to the number of the nodes.

$\mathrm{N}$ is the number of the paths of the network.

Due to the fact that the purpose is to find the number of paths from the first node to the last node, the $M_{1 n}$ entry is considered from any matrix.

$N=M_{1 n}{ }^{1}+M_{1 n}{ }^{2}+M_{1 n}{ }^{3}+\ldots+M_{1 n}{ }^{n}$

Which in this problem $\mathrm{n}=9$.

\section{2-2 The path production algorithm}

In this method, each path consist of a set integer numbers, which each number of this set has a node number of existing nodes. This method is based on the random numbers. Since any path starts from the first node $(X(1)=1)$, any random number is between the second and the last node. Thus to generate a path, the random number is created between the second node and the last one. Due to each path must be justifiable, in any stage before number entry in path and the random number must are related, the network matrix is studied. Any entry of this matrix is shown by $a_{i j}$. If $a_{i j}=1$ then, the random number will be fixed on the path; otherwise, another random number will be created. ( $\mathrm{i}=$ before number entry in path, $\mathrm{j}=$ the random number). Consequently, and like this, it continues to find all nodes on any path. As an example in the mentioned example, the first node in path is 1 , assume the created random number is 4 ; based on the project matrix, $a_{14}=0$ so the fourth node is not calculated (justifiable) and new random number is created. So justifiable paths are created along this method. The implementation of the proposed model of generate paths is shown In Fig 1. 


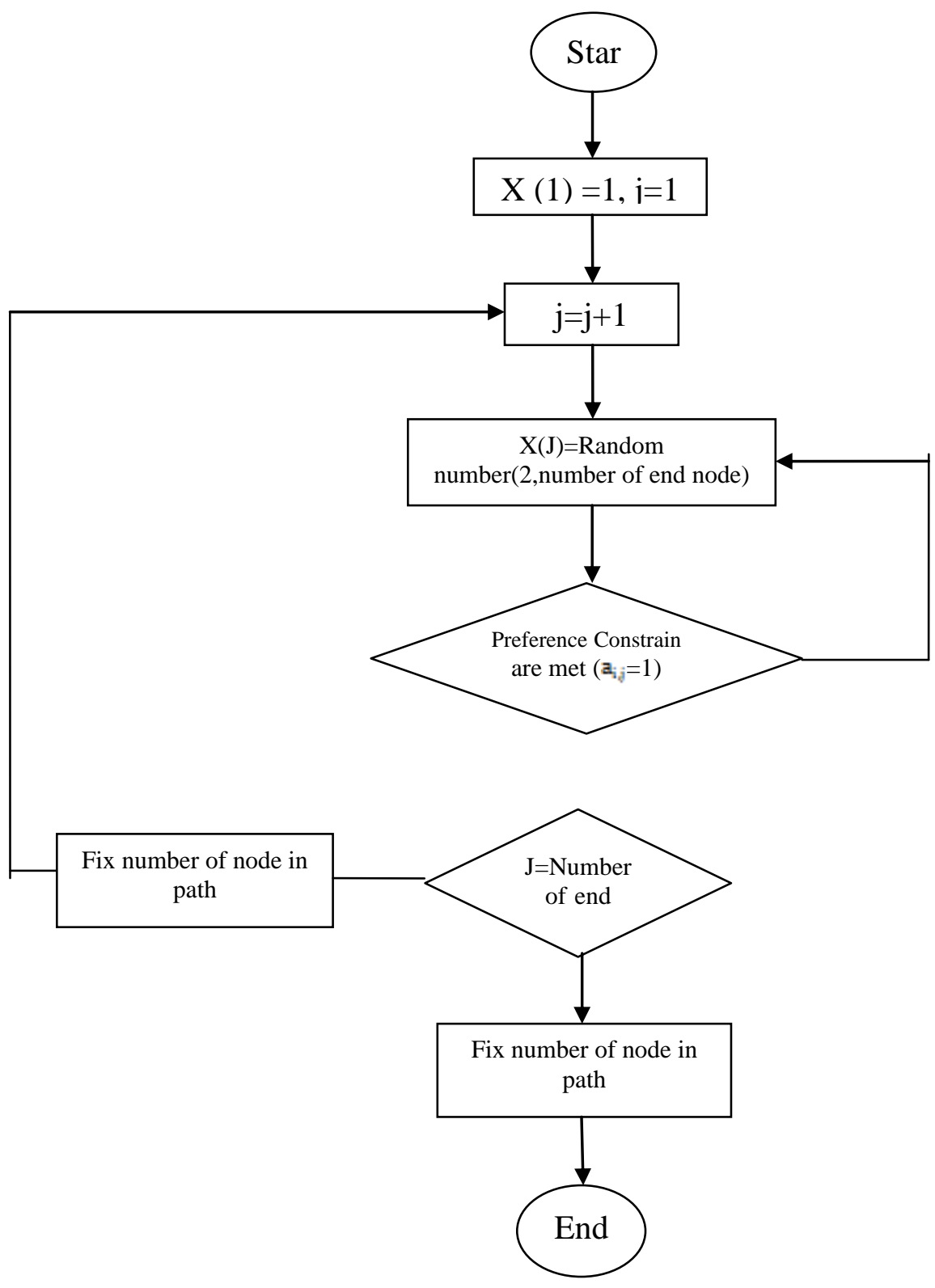

Fig.1.The proposed model of generate paths

2-3. The algorithm of the calculation of project critical path.

For implementation the algorithm below steps will done:

1-First, the data are read

2- The number of paths in the project network are calculated

3 - With the path production algorithm all of paths are generated

4- Completion time of each path are calculated. 
5-In final step with using Fuzzy theory the Critical Path is specified. In this section fuzzy number ranking is used in [6] will be explained.

The fuzzy number $\mathrm{u}$ is considered as $\mathrm{u}=\left(x_{0}, y_{0}, \alpha, \beta\right)$ which $x_{0}, y_{0}$ are two non-fuzzy numbers are considered with the left distance $\alpha>0$, the right distance $\beta>0$. therefore the fuzzy membership function $\mathrm{u}(\mathrm{x})$ is defined as:

$u(x)=\left\{\begin{array}{cc}\frac{1}{\alpha(x-x 0+\alpha)} & x 0-\alpha \leq x \leq x 0 \\ 1 & y 0 \leq x \leq y 0+\beta \\ \frac{1}{\beta(y 0-x+\beta)} & o . w\end{array}\right\}$

$U_{1}(r)=X_{0}-\alpha+\alpha_{r}, U_{2}(r)=Y_{0}+\beta-\beta_{r}$

In this method, to compare two fuzzy numbers the concept of distance has been used; and the name of this method is the method of D-distance. This distance will be calculated for both numbers, and any distance which is longer is larger.

$$
\text { For } a \in \mathrm{R}=\mathrm{Ua}(\mathrm{x})=\left\{\begin{array}{ll}
1 & x=a \\
0 & x \neq a
\end{array}\right\}
$$

If $\mathrm{a}=0$ then

$$
\text { For } a \in \mathrm{R}=\mathrm{U} 0(\mathrm{x})=\left\{\begin{array}{ll}
1 & x=a \\
0 & x \neq a
\end{array}\right\}
$$

The presented distance is calculated as:

$$
D(u, u 0)=\sqrt[2]{\int_{0}^{1}\left(\mathrm{u} 1(\mathrm{r})^{2}+\mathrm{u} 2(\mathrm{r})^{2}\right) \mathrm{dr}}
$$

For triangle fuzzy number $\mathrm{u}=\left(x_{0}, \alpha, \beta\right)$

$$
d(u, u 0)=\sqrt[2]{2 x 0^{2}+\left(\alpha^{2} / 3\right)+\left(\beta^{2} / 3\right)+x 0(\beta-\alpha)}
$$

For trapezoidal fuzzy number $\mathrm{u}=\left(x_{0}, y_{0}, \alpha, \beta\right)$

$$
d(u, u 0)=\sqrt[2]{2 x 0^{2}+\left(\alpha^{2} / 3\right)+\left(\beta^{2} / 3\right)-x 0 \alpha+y 0 \beta}
$$

Comparison between two fuzzy numbers $u$ and $a$ is as follow: 
For $u, a \in \mathrm{E}=\left\{\begin{array}{ll}u>a & d(u, u 0)>d(a, u 0) \\ u<a & d(u, u 0)<d(a, u 0) \\ u=a & d(u, u 0)=d(a, u 0)\end{array}\right\}$

And also the addition of two fuzzy numbers $\AA=\left(\mathrm{a}_{1}, \mathrm{a}_{2}, \alpha_{\mathrm{A}}, \beta_{\mathrm{A}}\right)$ and $B O=\left(\mathrm{b}_{1}, \mathrm{~b}_{2}, \alpha_{\mathrm{B}}, \beta_{\mathrm{B}}\right)$ is done as:

$A^{4}+B^{\prime}=\left(a_{1}+b_{1}, a_{2}+b_{2}, \alpha_{A}+\alpha_{B}, \beta_{A}+\beta_{B}\right)$

\section{Practical example}

To scrutinize the presented method, take a project with 12 activities which is as follows:

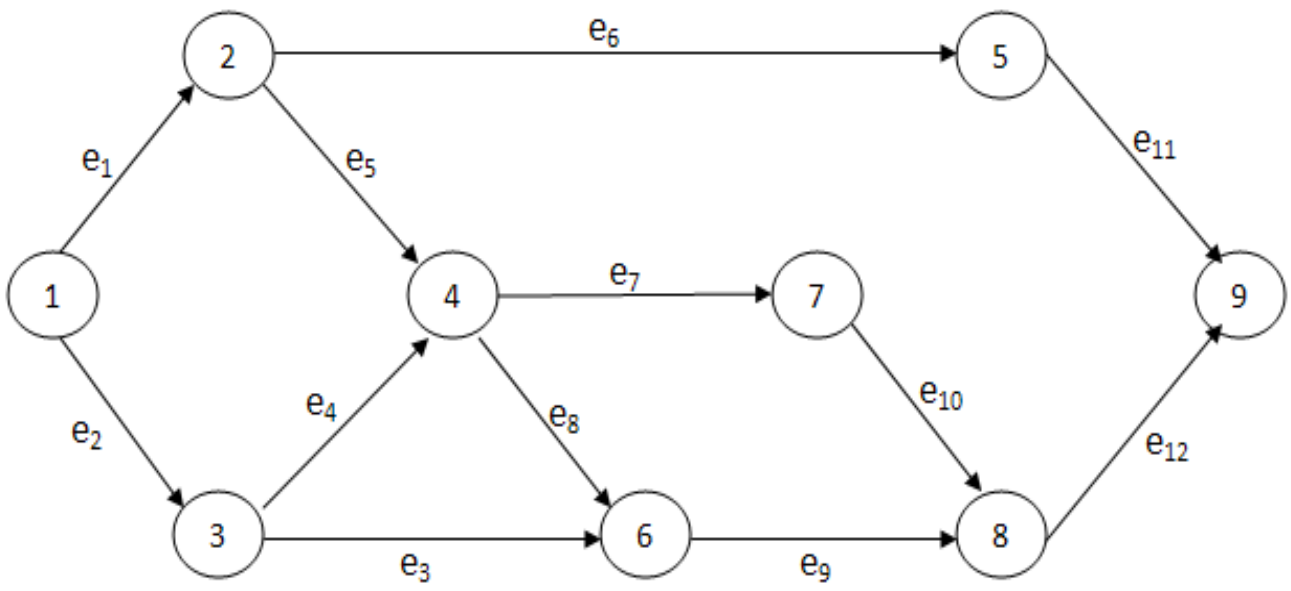

Fig.2. Project network

Each activity time is in the form of trapezoidal fuzzy numbers:

$$
\begin{aligned}
& P_{1}=(1,1.5,1,1), \ell_{2}=(2,3,0,2), \digamma_{3}=(6,7,0,2) \text {, } \\
& P_{4}=(0,0,0,0), P_{5}=(0,0,0,0), P_{6}=(2,3,1,2), \\
& q_{7}=(9,9,1,1), q_{8}=(5,5,1,1), \mathscr{Y}_{9}=(4,4,2,2) \text {, } \\
& \mathscr{\ell}_{10}=(3,4,2,0), \ell_{11}=(8,9,2,4), \ell_{12}=(6,9,2,3)
\end{aligned}
$$

And the network matrix is defined as: 


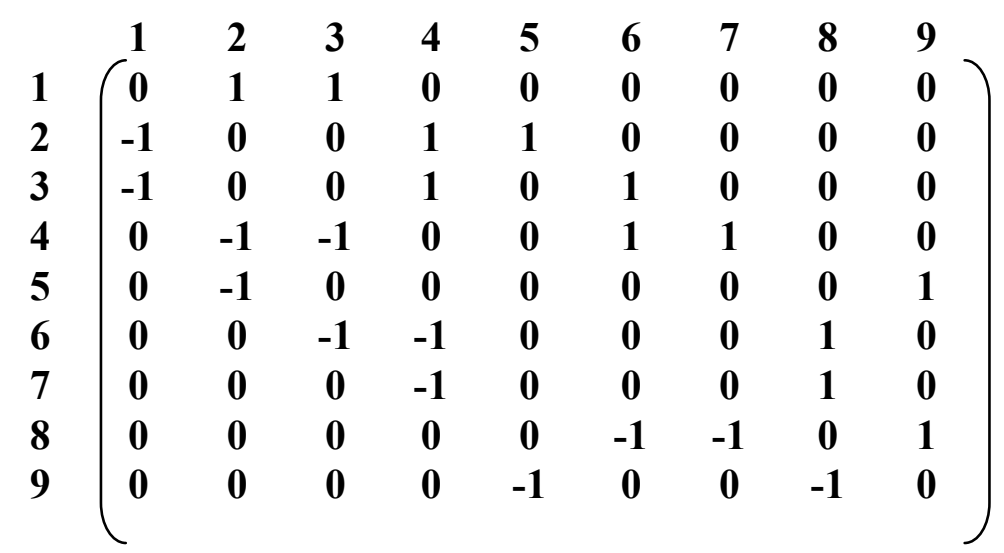

The problem is solved by Chanas \& Zieliński[1]; Shahsavari pour et al.[5]; Chen $\&$ Hsueh[7] and Shahsavari pour et al.[8] and the critical path is 1-3-4-7-8-9.

Table 1, the answer of Chanas solution

\begin{tabular}{|c|c|}
\hline$p \epsilon P$ & $\mu_{\beta 0}(p)$ \\
\hline $1---2---5---9$ & 0.6269 \\
$1---2---4---7---8---9$ & 0.5001 \\
$1---2---4---6---8---9$ & 0.3854 \\
$1---3---4---7---8---9$ & 1 \\
$1---3---4---6---8---9$ & 0.0001 \\
$1---3---6---8---9$ & 0.9941 \\
\hline
\end{tabular}

According to this method, paths are created and time of each path will be calculated. As the completion time of each activity is trapezoidal fuzzy number, each path time and the finally the critical path are too. After comparing among the created path, through the presented ranking method, the critical path is calculated that in the study it is $\{1-3-4-7-8-9\}$ with the time $(20,25,5,4)$. And it is clear in any method the same answer is reached; and the critical path is calculated in fuzzy condition.

\section{Conclusion}

For project to continue according to the predicted time and ends on time, the prediction must be made correctly. One of the important factors is to determines the activities time that most exact calculation is not possible. So it is necessary to determine the time with uncertainty. CPM could be very good tool for a manager to perform a project. In this study, a method to find the critical path will be presented. The advantage of this method is that unjustified paths won't be created. Also the time of activities will be calculated according to trapezoidal fuzzy number, and the calculations and completion time are too. To compare the fuzzy numbers, D- distance method is used. This method uses VBA in excel coding which leads to correct and exact result. Finally some other comparison will be done showing the same results. However, in this method the critical path is calculated with uncertainty, and the last number is a fuzzy number. 


\section{References}

[1] P. Chanas, Zielinski, Critical path analysis in the network with fuzzy activity times", Fuzzy Sets Syst, Vol. 122 (2001), pp. 195-204.

[2] D. Dubois, H. Fargier, V. Galvagnon, On latest starting times and floats in activity networks with ill-known durations", Eur. J. Oper.Res, Vol. 147 (2003), pp. 266-280.

[3] Zieliński, " On computing the latest starting times and floats of activities in a network with imprecise durations", Fuzzy Sets and Systems, Vol. 150 (2005), pp. 53-76.

[4] L. Feng-TES, Y. Jing-Shing, " Fuzzy critical path method based on singneddistance ranking and statistical confidence-interval estimates", The journal of supercomputing, Vol. 24,3 (2003), pp. 305-325 .

[5] Shahsavari pour, N., Modarres, M., Aryanejad, M. B.\& Tavakoli Moghadam, R." Calculating the project network critical path in uncertainty conditions", International Journal of Engineering and Technology, Vol. 2(2) (2010), pp. 136140 .

[6] Saeid Abbasbandy, "Ranking of fuzzy number, some recent and new formulas", IFSA-EUSFLAT, (2009).

[7] Chen,Shin-Pin. and Yi-Ju,Hsueh, A sample approach to fuzzy critical path analysis in project networks", Applied Mathematical Modeling, Vol. 32 (2008), pp. 1289-1297.

[8] N. Shahsavari Pour, M. Kheradmand, M. Fallah and S. Zeynali A new method for critical path method with fuzzy processing time", Management Science Letters 1 (2011). 